\title{
RESTRICTION OF LUNG VOLUMES BUT NORMAL PULMONARY TISSUE FUNCTION IN PATIENTS WITH MULIBREY NANISM
}

Johanna Sivunen$^{1}$, Merja Kajosaari ${ }^{1}$, Susann Karlberg${ }^{1}$, Marita Lipsanen-Nyman ${ }^{1}$, Hannu Jalanko ${ }^{1}$, Päivi Piirilä², Anssi Sovijärvi

${ }^{1}$ Children's Hospital, University of Helsinki and Helsinki University Hospital, Finland

2 Department of Clinical Physiology, University of Helsinki and Helsinki University Hospital, Finland

\section{BACKGROUND}

- Mulibrey nanism (MUL) is a rare growth restriction disorder with multiple organ manifestations.

- It is caused by genetic defects affecting TRIM37 protein.

- Perimyocardial heart disease is the most serious manifestation.

- Patients are short (adult height less than 3 SD below the population median), the thorax is small, and the extremities are slender.

- MUL patients often suffer from exercise-induced dyspnea, which can only partly be explained by the heart disease.

- Many MUL patients are treated with asthma medicines.

- Aim: to characterize the pulmonary pathophysiology of MUL.

Figure 1.

Bell-shaped thorax with

thin ribs in a

15-year old

MUL patient

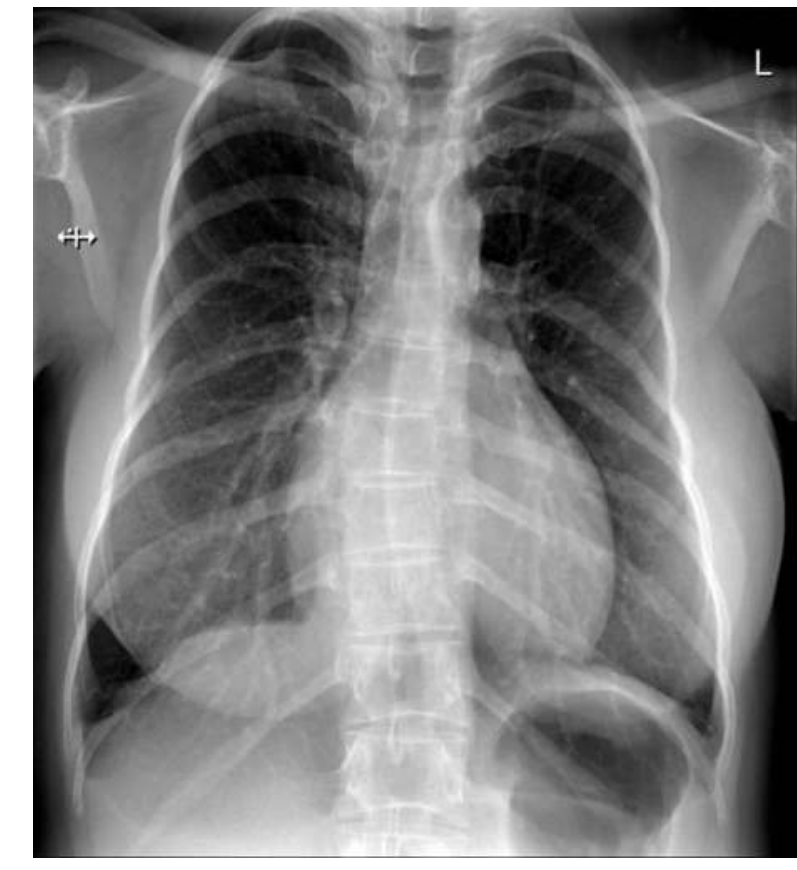

\section{METHODS}

- 33 Finnish MUL patients

- Lung function tests

- flow-volume spirometry with bronchodilatation test

- single-breath diffusing capacity for carbon monoxide

- single-breath lung volume measurements with helium dilution

- thoracic gas volume, airway resistance and specific conductance measurements with a body plethysmograph

- All variables were compared with predicted values and correlated with blood BNP

\section{RESULTS}

- Lung volumes (VC, FVC, TLCHe, TLCb) were markedly reduced $(45.1-53.6 \%$ of predicted, p mostly $<0.001)$.

- Reduced forced expiratory volume in the first second (FEV1, $\mathrm{p}<0.001,47-57 \%$ ).

- No signs of airway obstruction (normal FEV1/FVC, specific airway conductance SGaw and residual volume RV/TLC).

- Diffusing capacity (DLCO) was decreased ( $p<0.001,60-67 \%)$, but when related to alveolar volume (DLCO/VA), it was increased $(p<0.001,130-148 \%)$.

- Bronchodilatation suggesting asthma (dFEV1 >12\% and >200 ml) was found only in one patient.

- BNP correlated negatively with lung volumes.

\section{CONCLUSIONS}

- MUL patients typically have volume restriction of the lungs.

- Function of the pulmonary tissue remains intact.

- Airflow limitation is not typical.

- Asthma is a rare finding.

- The results suggest that in MUL, functionally normal lungs are stuffed into an abnormally small thoracic cage.
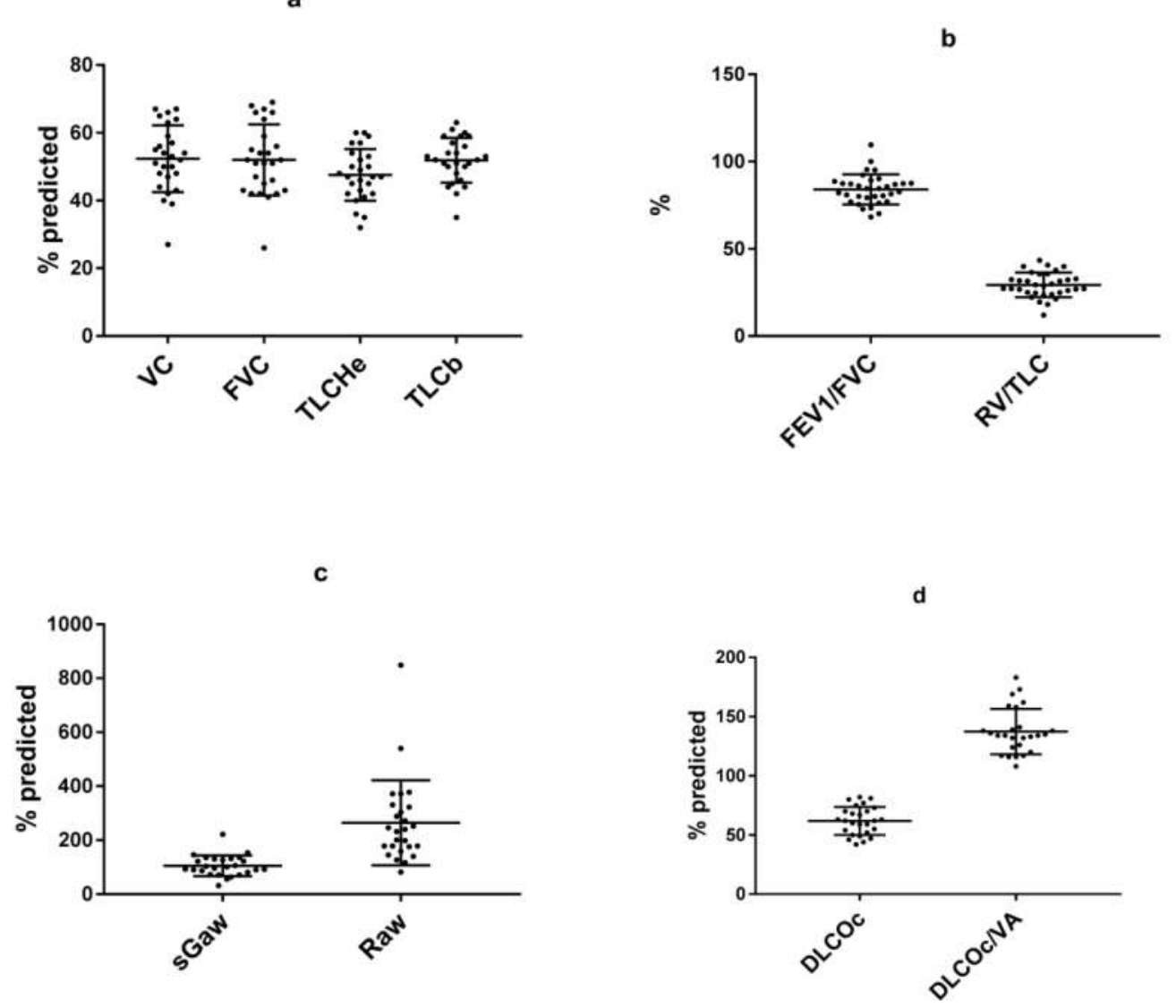

Figure 2. Individual lung values

a lung volume values VC, FVC, TLCHe and TLCb

b FEV1/FVC and RV/TLC

c Specific conductance (sGaw) and airway resistance (Raw)

d Diffusion capacity (DLCOc) and specific diffusion capacity (DLCOc/VA)

\begin{tabular}{|c|c|c|}
\hline & male & female \\
\hline$N(\%)$ & $17(52)$ & $16(49)$ \\
\hline age, years & $\begin{array}{l}25.05(15.93- \\
38.77)\end{array}$ & $29.03(15.21-49.96)$ \\
\hline weight, kg & $46.0(23.0-57.9)$ & $39.9(30.0-54.1)$ \\
\hline measured height, $\mathrm{cm}$ & $\begin{array}{c}152.5(137.0- \\
162.4)\end{array}$ & $137.7(130.0-152.0)$ \\
\hline estimated height ${ }^{\mathrm{a}}, \mathrm{cm}$ & $\begin{array}{c}161.5\left(\begin{array}{c}142.3-169.2 \\
n=15)\end{array}\right.\end{array}$ & $\begin{array}{c}144.0(134.0-161.3 \\
n=15)\end{array}$ \\
\hline $\mathrm{BMI}, \mathrm{kg} / \mathrm{m}^{2}$ & $19.8(12.3-23.6)$ & $21.1(16.8-28.2)$ \\
\hline $\begin{array}{l}\text { Steroid medication, } \mathrm{n} \\
(\%)\end{array}$ & $0(0)$ & $4(25)$ \\
\hline Current smoker, n (\%) & $3(18)$ & $2(13)$ \\
\hline $\begin{array}{l}\text { Diastolic dysfunction in } \\
\text { ECG, } n(\%)\end{array}$ & $7(41)$ & $4(25)$ \\
\hline $\begin{array}{l}\text { Pericardiectomy } \\
\text { performed earlier, n (\%) }\end{array}$ & $9(53)$ & $4(25)$ \\
\hline BNP, ng/Lb & $9.3(4.0-90.0)$ & $23.0(9.1-71.0)$ \\
\hline
\end{tabular}

Values are in median (range) unless else specified aestimated total height derived from sitting height bnormal value $<18.7 \mathrm{ng} / \mathrm{L}$ 